\title{
ALMA OBSERVATIONS OF WARM MOLECULAR GAS AND COLD DUST IN NGC 34*
}

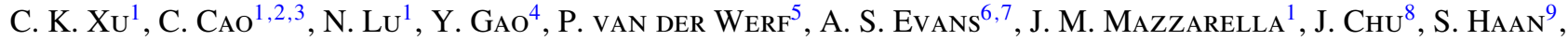 \\ T. DiaZ-Santos ${ }^{1}$, R. MeiJerinK ${ }^{5}$, Y.-H. ZhaO ${ }^{1,4}$, P. ApPleton ${ }^{1}$, L. Armus ${ }^{1}$, V. Charmandaris ${ }^{10,11,12}$, S. LORD $^{1}$, \\ E. J. Murphy ${ }^{1}$, D. B. SAnders ${ }^{8}$, B. Schulz ${ }^{1}$, And S. Stierwalt ${ }^{6,13}$ \\ ${ }^{1}$ Infrared Processing and Analysis Center, MS 100-22, California Institute of Technology, Pasadena, CA 91125, USA \\ ${ }^{2}$ School of Space Science and Physics, Shandong University at Weihai, Weihai, Shandong 264209, China; caochen@ sdu.edu.cn \\ ${ }^{3}$ Shandong Provincial Key Laboratory of Optical Astronomy and Solar-Terrestrial Environment, Weihai, Shandong 264209, China \\ ${ }^{4}$ Purple Mountain Observatory, Chinese Academy of Sciences, 2 West Beijing Road, Nanjing 210008, China \\ ${ }^{5}$ Leiden Observatory, Leiden University, P.O. Box 9513, NL-2300 RA Leiden, Netherlands \\ ${ }^{6}$ Department of Astronomy, University of Virginia, P.O. Box 400325, Charlottesville, VA 22904, USA \\ ${ }^{7}$ National Radio Astronomy Observatory, Charlottesville, VA 22904, USA \\ ${ }^{8}$ Institute for Astronomy, University of Hawaii, 2680 Woodlawn Drive, Honolulu, HI 96816, USA \\ ${ }^{9}$ CSIRO Astronomy and Space Science, ATNF, P.O. Box 76, Epping 1710, Australia \\ ${ }^{10}$ Department of Physics, University of Crete, GR-71003, Heraklion, Greece \\ ${ }^{11}$ Institute for Astronomy, Astrophysics, Space Applications \& Remote Sensing, \\ National Observatory of Athens, GR-15236, Penteli, Greece \\ ${ }^{12}$ Chercheur Associé, Observatoire de Paris, F-75014, Paris, France \\ ${ }^{13}$ Spitzer Science Center, California Institute of Technology, 1200 E. California Boulevard, Pasadena, CA 91125, USA \\ Received 2013 November 25; accepted 2014 April 3; published 2014 May 2
}

\begin{abstract}
We present Atacama Large Millimeter Array (ALMA) Cycle-0 observations of the CO (6-5) line emission (restframe frequency $=691.473 \mathrm{GHz}$ ) and of the $435 \mu \mathrm{m}$ dust continuum emission in the nuclear region of NGC 34, a local luminous infrared galaxy at a distance of $84 \mathrm{Mpc}\left(1^{\prime \prime}=407 \mathrm{pc}\right)$ which contains a Seyfert 2 active galactic nucleus (AGN) and a nuclear starburst. The $\mathrm{CO}$ emission is well resolved by the ALMA beam $(0,26 \times 0$ '.23), with an integrated flux of $f_{\mathrm{CO}(6-5)}=1004( \pm 151) \mathrm{Jy} \mathrm{km} \mathrm{s}^{-1}$. Both the morphology and kinematics of the $\mathrm{CO}(6-5)$ emission are rather regular, consistent with a compact rotating disk with a size of $200 \mathrm{pc}$. A significant emission feature is detected on the redshifted wing of the line profile at the frequency of the $\mathrm{H}^{13} \mathrm{CN}$ (8-7) line, with an integrated flux of $17.7 \pm 2.1$ (random) \pm 2.7 (systematic) $\mathrm{Jy} \mathrm{km} \mathrm{s}^{-1}$. However, it cannot be ruled out that the feature is due to an outflow of warm dense gas with a mean velocity of $400 \mathrm{~km} \mathrm{~s}^{-1}$. The continuum is resolved into an elongated configuration, and the observed flux corresponds to a dust mass of $M_{\text {dust }}=10^{6.97 \pm 0.13} M_{\odot}$. An unresolved central core (radius $\simeq 50 \mathrm{pc}$ ) contributes $28 \%$ of the continuum flux and $19 \%$ of the $\mathrm{CO}(6-5)$ flux, consistent with insignificant contributions of the AGN to both emissions. Both the $\mathrm{CO}(6-5)$ and continuum spatial distributions suggest a very high gas column density $\left(\gtrsim 10^{4} M_{\odot} \mathrm{pc}^{-2}\right)$ in the nuclear region at radius $\lesssim 100 \mathrm{pc}$.
\end{abstract}

Key words: galaxies: active - galaxies: evolution - galaxies: general - galaxies: interactions - galaxies: starburst galaxies: Seyfert - submillimeter: galaxies

\section{INTRODUCTION}

Luminous infrared galaxies (LIRGs; $L_{\mathrm{IR}}[8-1000 \mu \mathrm{m}]>$ $10^{11} L_{\odot}$ ), including ultra-luminous infrared galaxies (ULIRGs; $L_{\mathrm{IR}}>10^{12} L_{\odot}$ ), have a space density exceeding that of optically selected starburst and Seyfert galaxies at comparable bolometric luminosity (Soifer et al. 1987). They are an ensemble of single galaxies, galaxy pairs, interacting systems, and advanced mergers (Sanders \& Mirabel 1996; Wang et al. 2006). Most (U)LIRGs of $L_{\mathrm{IR}} \gtrsim 10^{11.5} L_{\odot}$ are advanced mergers (including merger remnants) harboring extreme starbursts (star formation rate (SFR) $\gtrsim 50 M_{\odot} \mathrm{yr}^{-1}$ ) and powerful active galactic nuclei (AGNs; Kim et al. 2002). Toomre (1977) was the first to suggest that merging can transform spirals into ellipticals, a theory that has been borne out observationally (Schweizer 1982; Genzel et al. 2001). Strong outflows of neutral and ionized gas were widely detected among (U)LIRGs (Armus et al. 1990; Heckman et al. 1990; Rupke et al. 2005). Recently, massive molecular gas outflows have been found in (U)LIRGs with powerful AGNs (Fischer et al. 2010; Feruglio et al. 2013, 2010;

* The National Radio Astronomy Observatory is a facility of the National Science Foundation
Universities, Inc.
Sturm et al. 2011; Aalto et al. 2012; Veilleux et al. 2013; Combes et al. 2013; Cicone et al. 2014). Hence, feedback from merger induced extreme starbursts and AGNs is the most popular mechanism for explaining the star formation quenching in massive galaxies that lead to the formation of red-sequence galaxies (Faber et al. 2007; Hopkins et al. 2008a), and (U)LIRGs are the best local laboratories for studying these processes. However, due to the enormous dust obscuration in (U)LIRGs (Sanders \& Mirabel 1996), it is very difficult to study them using high angular resolution optical/NIR instruments. Observations using space FIR/submillimeter observatories, such as Spitzer (Werner et al. 2004) and Herschel (Pilbratt et al. 2010), can penetrate the dust obscuration. However, their angular resolutions ( a few arcsecs) are not sufficient to resolve the nuclei in most (U)LIRGs. The Atacama Large Millimeter Array (ALMA; Wootten \& Thompson 2009) is changing the situation rapidly. Once completed, submillimeter/millimeter observations using the ALMA full array (66 antennas) will detect both the line emission of gas and the continuum emission of dust (heated either by starburst or AGN, or both) with an angular resolution of $\lesssim 0.1^{\prime \prime}$, revealing interplays between gas, starbursts, and AGNs in (U)LIRG nuclei down to linear scales of $\sim 10 \mathrm{pc}$ in the nearest systems. 
Table 1

ALMA Observations

\begin{tabular}{lccccccc}
\hline \hline SB & $\begin{array}{c}\text { Date } \\
(\text { yyyy/mm/dd) }\end{array}$ & $\begin{array}{c}\text { Time } \\
(\mathrm{UTC})\end{array}$ & Config & $N_{\text {ant }}$ & $\begin{array}{c}l_{\max } \\
(\mathrm{m})\end{array}$ & $\begin{array}{c}t_{\text {int }} \\
(\mathrm{min})\end{array}$ & $\begin{array}{r}T_{\text {sys }} \\
(\mathrm{K})\end{array}$ \\
& $(2)$ & $(3)$ & $(4)$ & $(5)$ & $(6)$ & $(7)$ & $(8)$ \\
\hline X40e374_Xba3 & $2012 / 5 / 20$ & $09: 17: 15-10: 35: 34$ & $\mathrm{E}$ & 16 & 375 & 24.7 & 850 \\
X40e374_Xd36 & $2012 / 5 / 20$ & $10: 48: 14-12: 06: 33$ & $\mathrm{E}$ & 16 & 375 & 24.7 & 653 \\
X40e374_Xec9 & $2012 / 5 / 20$ & $12: 22: 31-13: 07: 05$ & $\mathrm{E}$ & 16 & 375 & 9.9 & 654 \\
X41065b_X334 & $2012 / 5 / 21$ & $09: 47: 04-11: 06: 00$ & $\mathrm{E}$ & 16 & 375 & 24.7 & 634 \\
X41065b_X4c7 & $2012 / 5 / 21$ & $11: 20: 00-10: 41: 09$ & $\mathrm{E}$ & 16 & 375 & 24.7 & 528 \\
X4afc39_X43b & $2012 / 8 / 25$ & $03: 30: 38-05: 01: 41$ & $\mathrm{E}$ and C & 27 & 402 & 24.7 & 1058 \\
\hline
\end{tabular}

Notes. Column 1: schedule-block number; 2 and 3: observation date and time; 4: configuration; 5: number of antennas; 6: maximum baseline length; 7: on-target integration time; 8: median $T_{\text {sys }}$.

In this paper, we report ALMA Cycle-0 observations (utilizing up to 27 antennas) of the CO (6-5) line (rest-frame frequency $=691.473 \mathrm{GHz}$ ) emission and of $435 \mu \mathrm{m}$ dust continuum emission in the nuclear region of NGC 34 , a local LIRG $\left(L_{\mathrm{IR}}=10^{11.49} L_{\odot}\right.$; Armus et al. 2009) containing an Sy2 AGN and a nuclear starburst (see Schweizer \& Seitzer 2007 for an overview). With an excitation temperature of $T_{\mathrm{ex}}=116.2 \mathrm{~K}$ and a critical density of $n_{\text {crit }}=2.9 \times 10^{5} \mathrm{~cm}^{-3}$ (Carilli \& Walter 2013), the CO (6-5) line probes the warm and dense gas that is much more closely related to the star formation activities than the cold and lower density gas probed by low $J$ CO lines (e.g., $\mathrm{CO}(1-0)$ and $\mathrm{CO}(2-1)$ ) commonly studied in the literature. Among the complete sample of 202 LIRGs of the Great Observatories All-sky LIRG Survey (GOALS; Armus et al. 2009), which were selected from the IRAS Revised Bright Galaxy Sample (Sanders et al. 2003), NGC 34 (also known as NGC 17, Mrk 938, and IRAS F00085-1223) was chosen for early ALMA observations because of the following features. (1) Among LIRGs that have the $\mathrm{CO}(6-5)$ line flux $f_{\mathrm{CO}(6-5)} \geqslant 1000 \mathrm{Jy} \mathrm{km} \mathrm{s}^{-1}$ observed in the Herschel SPIRE Fourier Transform Spectrometer (FTS) survey of GOALS galaxies (angular resolution: $\sim 30^{\prime \prime}$; P. van der Werf et al., in preparation; Lu et al. 2014), it is one of the closest with a distance of $D=84 \mathrm{Mpc}\left(1^{\prime \prime}=407 \mathrm{pc}\right)$. This enables high signal-to-noise ratio observations of warm gas structures with the best linear resolution for a given angular resolution. (2) With a declination of $-12^{\circ}$, NGC 34 transits at $\sim 11^{\circ}$ from the zenith, therefore its Band 9 observations are affected by minimal atmospheric absorption. (3) The Keck-MIRLIN images of Gorjian et al. (2004) detected $\gtrsim 50 \%$ of the $12 \mu \mathrm{m}$ IRAS flux in the central $\sim 1 \mathrm{kpc}$ region of NGC 34, indicating strong nuclear star formation and/or AGN activities.

Early observations of the $\mathrm{CO}(6-5)$ emission in nearby starburst galaxies NGC 253, IC 342, and M 82 (Harris et al. 1991) showed that the molecular gas in starbursts is warmer than in normal disk galaxies. Papadopoulos et al. (2012) carried out an extensive survey for higher $J \mathrm{CO}$ lines, including the $\mathrm{CO}$ (6-5) line, for LIRGs using JCMT and found many of them have the global $\mathrm{CO}$ spectral line energy distribution dominated by a very warm $(T \sim 100 \mathrm{~K})$ and dense $\left(n \geqslant 10^{4} \mathrm{~cm}^{-3}\right)$ gas phase. The CO (6-5) map of the central region of Arp 220 obtained by Matsushita et al. (2009) using the SMA has an angular resolution of $\sim 1^{\prime \prime}(\sim 400 \mathrm{pc})$, revealing two warm gas components associated with the two nuclei (separation $\sim 400 \mathrm{pc}$ ), but the data were unable to resolve the individual components. The SMA observations of the CO (6-5) line emission of VV 114 (Sliwa et al. 2013) has a relatively coarse resolution of 2 ".5 ( $\gtrsim 1 \mathrm{kpc})$. The new ALMA observations reported here, with an angular resolution of $\sim 0$ '.25, resolved the warm and dense gas in the nuclear region of an LIRG with a linear resolution of $\sim 100 \mathrm{pc}$ for the first time. We aim to answer the following questions with these observations: (1) how is the warm dense molecular gas distributed in the inner most region of NGC 34? (2) How is the gas related to the starburst and the AGN, respectively? (3) Is the dust emission dominated by an extended component, or by an unresolved compact component? (4) Is there evidence for a molecular outflow? Comparing with the extensive literature on NGC 34 (a late stage major-merger; Mazzarella \& Boroson 1993; Schweizer \& Seitzer 2007) and with high-resolution simulations (Hopkins et al. 2013), our results shed new light on how various activities and their feedback set the stage for a major-merger remnant to become a red-and-dead elliptical galaxy (Zubovas \& King 2012).

\section{OBSERVATIONS}

Observations of the CO (6-5) line emission and $435 \mu \mathrm{m}$ dust continuum emission in the nuclear region of NGC 34 were carried out using the Band 9 receivers of ALMA in the time division mode (velocity resolution: $6.8 \mathrm{~km} \mathrm{~s}^{-1}$ ). The four basebands (i.e., "Spectral Windows," hereafter SPWs) are centered at the sky frequencies of 679.801, 678.039, 676.271, and $674.339 \mathrm{GHz}$, respectively, each with a bandwidth of $\sim 2 \mathrm{GHz}$. Observations were carried out in both extended (E) and extend and compact (E and C) configurations (Table 1). The total on-target integration time was $2.25 \mathrm{hr}$. During the observations, phase and gain variations were monitored using QSOs 2348-165 and $3 \mathrm{C}$ 454.3. Observations of the asteroid Pallas were made for flux calibration. The error in the flux calibration was estimated to be $15 \%$.

The data were reduced and calibrated using CASA 3.4. The visibilities were imaged with natural weighting and cleaned. Finally, phase and amplitude self-calibrations were carried out to improve the overall sensitivity. Two data sets were generated from the observations. In the first data set, the $\mathrm{CO}(6-5)$ line data cube was generated using only data in SPW-0 (sky-freq = $678.039 \pm 1 \mathrm{GHz}$ ), which encompass the $\mathrm{CO}(6-5)$ line emission with an effective bandpass of $\sim 800 \mathrm{~km} \mathrm{~s}^{-1}$. The continuum was estimated using data in the other three SPWs. In the second data set, the $\mathrm{CO}(6-5)$ line data cube was generated using three SPWs: SPW-0, SPW-1, and SPW-3, centered at 678.039, 679.801 , and $676.271 \mathrm{GHz}$, respectively. The bandwidth of this CO (6-5) line data cube is $\sim 2400 \mathrm{~km} \mathrm{~s}^{-1}$, sufficient for the search of high velocity outflows. For the second data set, the continuum was estimated using only data in SPW-2 (sky-freq = $674.339 \pm 1 \mathrm{GHz}$ ). The first data set is preferred when there is no 


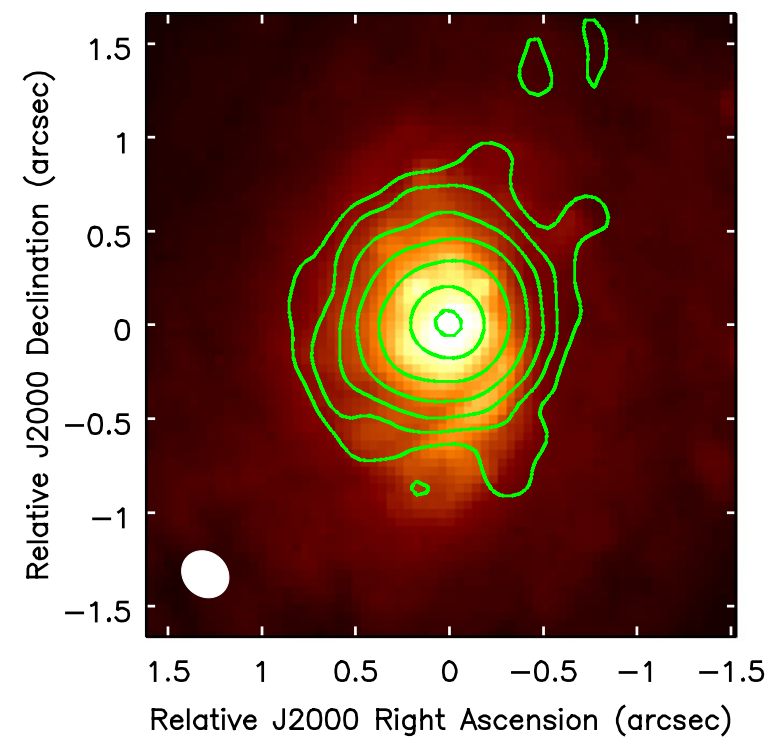

Figure 1. Contours of the integrated CO (6-5) line emission of NGC 34 on the HST $V$-band image (Malken et al. 1998). The contour levels are [1, 2, 4, 8, 16, $32,50] \times 3.6 \mathrm{Jy} \mathrm{beam}^{-1} \mathrm{~km} \mathrm{~s}^{-1}$. The white ellipse shows the synthesized beam size $\left(\mathrm{FWHM}=0^{\prime \prime} .26 \times 0.23\right.$, P.A. $\left.=46.3\right)$. The map center is at R.A.(J2000) $=00^{\mathrm{h}} 11^{\mathrm{m}} 6.537$ and decl.(J2000) $=-12^{\circ} 06^{\prime} 27^{\prime \prime} .49$.

significant evidence for outflows of $\delta v \gtrsim 400 \mathrm{~km} \mathrm{~s}^{-1}$ because of better continuum estimation and subtraction. The continuum subtraction was carried out using CASA task UVcontsub.

In order to increase the signal-to-noise ratio, we binned the spectral cubes into channels with the width of $\delta v=34 \mathrm{~km} \mathrm{~s}^{-1}$. The primary beam of the Band 9 observations is $\sim 8^{\prime \prime}$. For the configurations used in our observations, the resulting maps have good quality only in the central region of $\sim 3^{\prime \prime}$ (limited by the uv-coverage). Within this region, channel maps of the $\mathrm{CO}(6-5)$ line emission have $1 \sigma \mathrm{rms}$ noise of $5.5 \mathrm{mJy}^{\text {beam }}{ }^{-1}$. The rms of the integrated line emission map is $1.2 \mathrm{Jy}^{\text {beam }}{ }^{-1} \mathrm{~km} \mathrm{~s}^{-1}$, and the rms of the continuum map is $1.9 \mathrm{mJy} \mathrm{beam}^{-1}$. The synthesized beams of these maps are nearly identical, having the FWHM of $0{ }^{\prime \prime} 26 \times 0$.'23 (corresponding to physical scales of $106 \mathrm{pc} \times 94 \mathrm{pc}$ ) and the P.A. of 46.3. The absolute pointing accuracy of these ALMA observations is on the order of $0{ }^{\prime \prime} 1$.

\section{RESULTS}

\section{1. $C O$ (6-5) Line Emission}

The CO (6-5) line emission in the central region of NGC 34 (Figure 1$)$ is in a disk that extends to a radius of $0^{\prime \prime} 8(\sim 320 \mathrm{pc})$, as shown by the $3 \sigma$ contour. The disk is well resolved by the ALMA beam (Figure 2), with an FWHM of 0.'5 (200 pc). It fully covers the "central disk" of stars (semi-major axis $a \simeq 0^{\prime \prime} .8$, P.A. $\left.=4^{\circ}\right)$ which in turn is inside a larger $\left(\sim 8^{\prime \prime}\right)$ "exponential disk," both found in the Hubble Space Telescope (HST) $V$-band image by Schweizer \& Seitzer (2007). The $V$-band disk has an axial ratio of $b / a \simeq 0.72$, corresponding to an inclination angle of $i \simeq 44^{\circ}$. The CO (6-5) disk looks more face-on $(b / a \sim 0.9)$, though this could be affected significantly by beam smearing effects. The integrated line emission has a peak surface density of $196 \pm 29 \mathrm{Jy} \mathrm{km} \mathrm{s}^{-1}$ beam $^{-1}$ (peak brightness temperature $=36.8 \pm 5.5 \mathrm{~K}$ ) and a total flux of $f_{\mathrm{CO}(6-5)}=1004 \pm 151 \mathrm{Jy} \mathrm{km} \mathrm{s}^{-1}$. The uncertainties are dominated by the flux calibration error. This is consistent with the Herschel FTS measurement of the integrated CO (6-5) line emission of NGC 34, which is $937 \pm 63 \mathrm{Jy} \mathrm{km} \mathrm{s}^{-1}$ ( $\mathrm{Lu}$ et al.

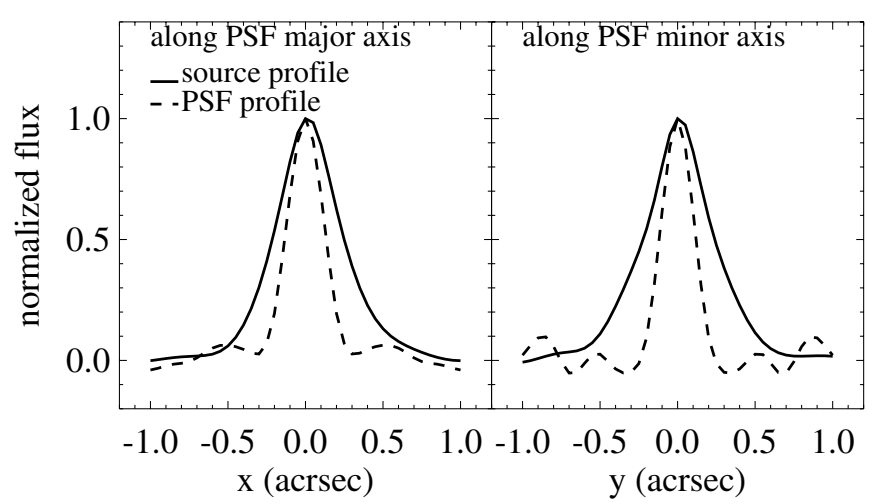

Figure 2. Comparisons of profiles of the integrated $\mathrm{CO}(6-5)$ line emission and the point-spread function (PSF) of the synthesized beam (FWHM $=00^{\prime \prime} .26 \times 0$.'23, P.A. $=46.3$ )

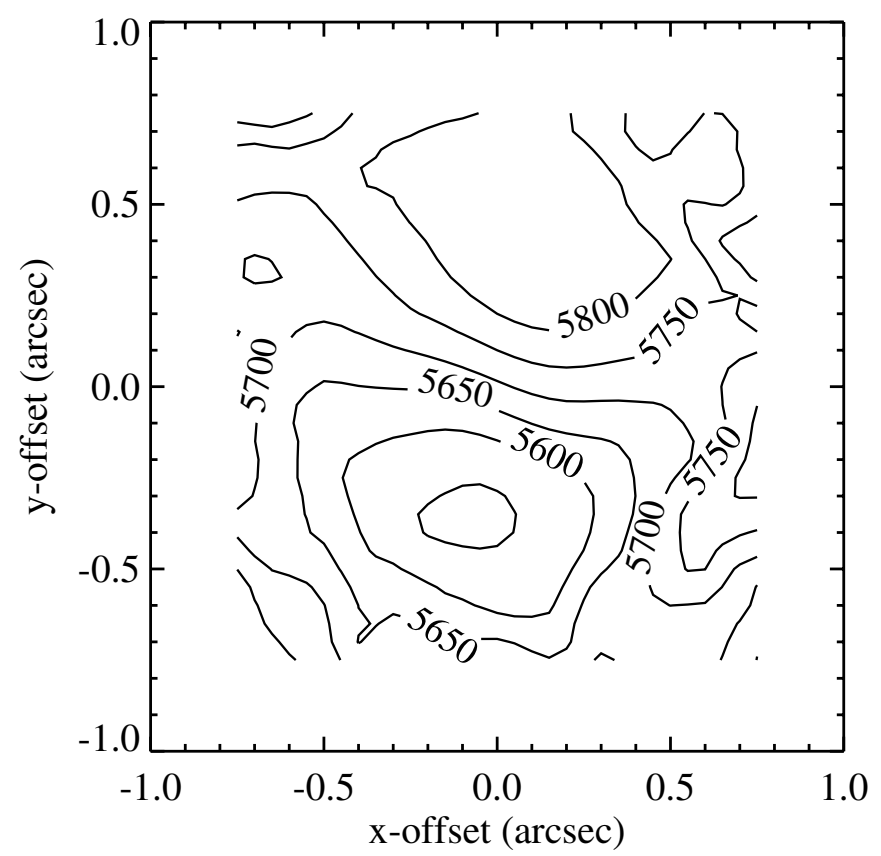

Figure 3. Contour map of the first moment. The LSR (radio) velocity contour levels are in the units of $\mathrm{km} \mathrm{s}^{-1}$.

2014), indicating that all of the warm molecular gas in the galaxy is concentrated in the central disk detected by ALMA.

The first-moment map is shown in Figure 3, and the channel maps are in Figure 4. A significant velocity gradient along the axis of P.A. $=345^{\circ}$ can be seen in these maps, consistent with a slightly inclined rotating disk. Figure 5 is a $P-V$ plot of the $\mathrm{CO}(6-5)$ line emission over a stripe of width $=00^{\prime \prime} 25$ and P.A. $=345^{\circ}$, passing through the central core. It shows that the rotation velocity first rises steadily in the inner part of the disk and then becomes quite flat at radius $\gtrsim 0$ '.5, again consistent with a resolved rotating gas disk that is mostly confined to radius $\lesssim 0^{\prime \prime} .5$.

Panel (a) of Figure 6 presents the spectrum of the CO (6-5) line emission in the radio heliocentric velocity domain, measured in the channel maps with an aperture of radius $=1^{\prime \prime}$. The double-horn shaped spectrum is again consistent with a rotating disk. The flux-weighted mean, a measure of the systematic velocity of the kinematic center of the warm molecular gas disk, is $5700 \mathrm{~km} \mathrm{~s}^{-1}$ with an measurement error of $\simeq 7 \mathrm{~km} \mathrm{~s}^{-1}$ set by the velocity resolution of the ALMA observations. The FWHM of the spectrum is $406 \mathrm{~km} \mathrm{~s}^{-1}$. 

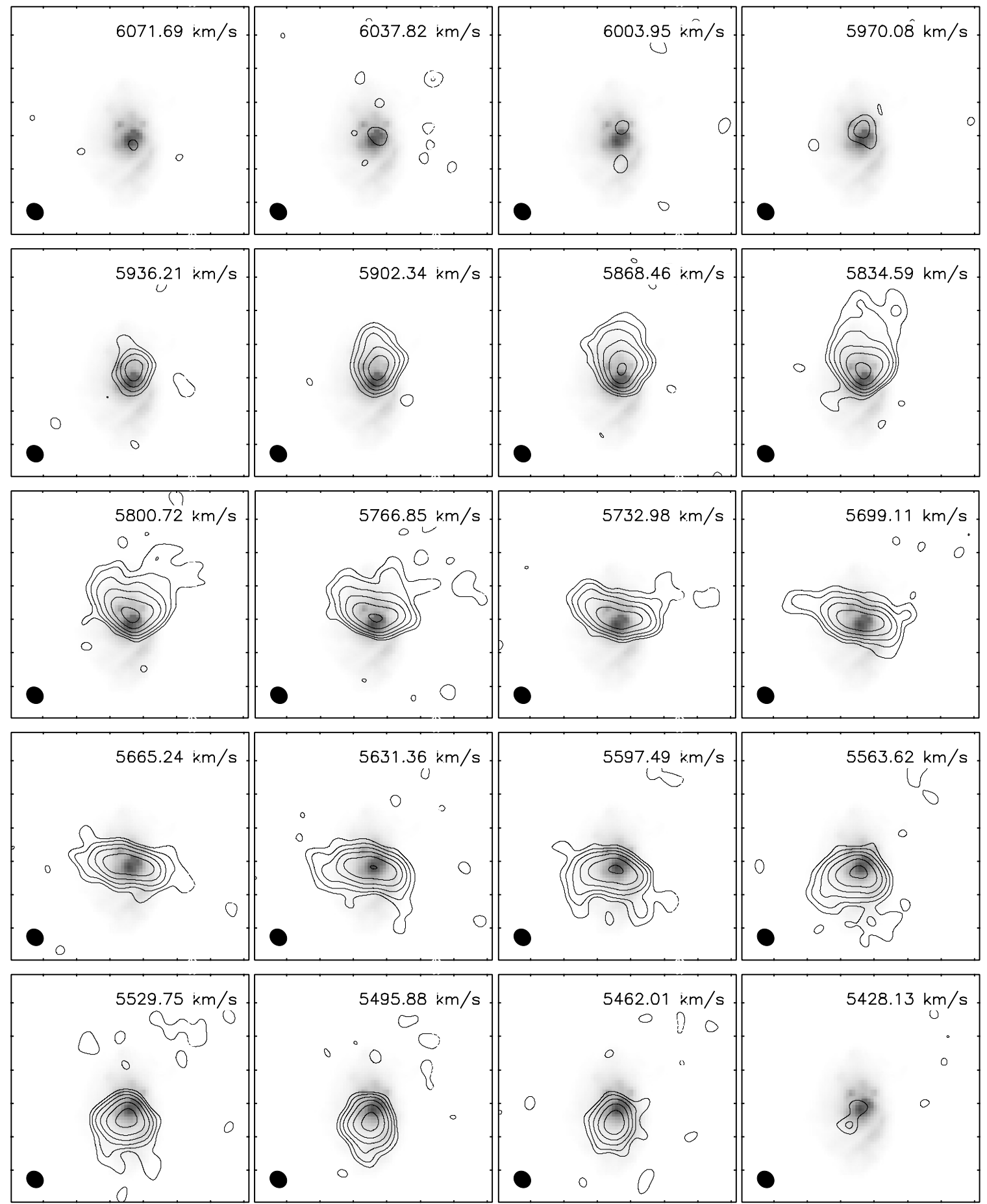

Figure 4. $\mathrm{CO}(6-5)$ line emission contours of the channel maps (velocity channel width $=34 \mathrm{~km} \mathrm{~s}^{-1}$ ), overlaid on the $H S T V$-band image. The contour levels are $16.5 \mathrm{mJy}$ beam $^{-1} \times[1,2,4,8,16,24]$. All maps have the same size of 3'.4 $\times 33^{\prime \prime} 4$ and the tick marks are separated by $0^{\prime \prime} 5$. In each channel, the central LSR (radio) velocity is given.

A significant emission feature is detected in the redshifted wing in the velocity range of $6000 \mathrm{~km} \mathrm{~s}^{-1} \lesssim v \lesssim 6200 \mathrm{~km} \mathrm{~s}^{-1}$ (panels (b) and (d) of Figure 6). The integrated flux (between $6000 \leqslant v \leqslant 6200 \mathrm{~km} \mathrm{~s}^{-1}$ ) is $17.7 \pm 2.1 \pm 2.7 \mathrm{Jy} \mathrm{km} \mathrm{s}^{-1}$ (the first error is due to rms noise and the second due to calibration error). The contour map of this feature is shown in Figure 7.

There are two possibilities for the cause of this feature. One is $\mathrm{H}^{13} \mathrm{CN}$ (8-7) line emission (rest-frame frequency = $690.552 \mathrm{GHz}$ ), a very high critical density isotopic molecular line $\left(n_{\text {crit }}=1.8 \times 10^{9} \mathrm{~cm}^{-3}\right.$ and $\left.T_{\text {ex }}=153 \mathrm{~K}\right)$. In this scenario, the integrated flux of the feature corresponds to a $f_{\mathrm{H}^{13} \mathrm{CN}(8-7)}$-to- $f_{\mathrm{CO}(6-5)}$ ratio of $0.018 \pm 0.002$. And the location of the peak of the feature corresponds to $v=5745 \mathrm{~km} \mathrm{~s}^{-1}$ for the $\mathrm{H}^{13} \mathrm{CN}(8-7)$ line, in very good agreement with the mean velocity of the $\mathrm{CO}(6-5)$ line emission (see Figure 6). While no detection of $\mathrm{H}^{13} \mathrm{CN}(8-7)$ has been reported previously for any extragalactic source, a few detections of lower $J \mathrm{H}^{13} \mathrm{CN}$ lines in nearby galaxies can be found in the literature (Mauersberger 


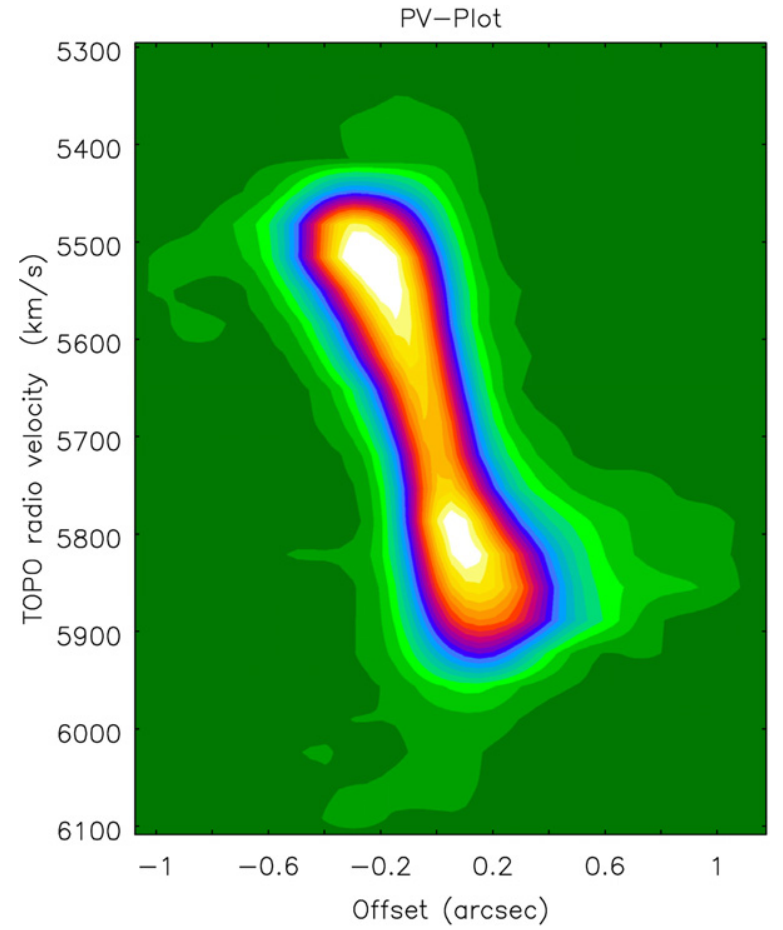

Figure 5. $P-V$ plot of the $\mathrm{CO}(6-5)$ line emission over a stripe (with the width of 0.25 and P.A. $=345^{\circ}$ ) passing through the central core.

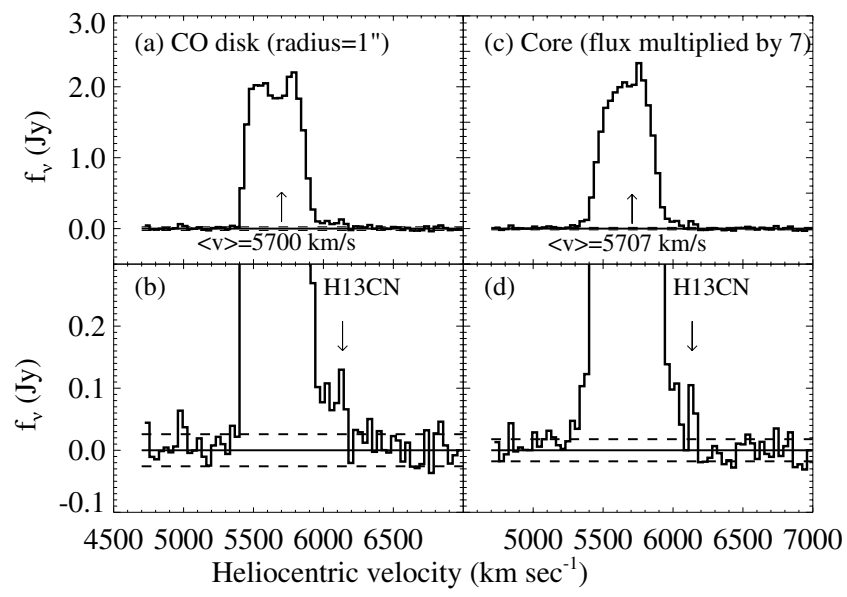

Figure 6. Panel (a): spectrum of the $\mathrm{CO}(6-5)$ line emission in radio heliocentric velocity domain, measured in the channel maps with an aperture of radius $=$ $1^{\prime \prime}=407 \mathrm{pc}$. The dashed lines mark the $1 \sigma \mathrm{rms}$ boundaries, which are $\pm 26 \mathrm{mJy} \times 34 \mathrm{~km} \mathrm{~s}^{-1}$. Panel (b): zoom-in plot of the bottom part of panel (a). Again the dashed lines mark the $1 \sigma \mathrm{rms}$ boundaries. The arrow marks the location of the $\mathrm{H}^{13} \mathrm{CN}(8-7)$ line with the velocity $v=5745 \mathrm{~km} \mathrm{~s}^{-1}$. Panel (c): spectrum of the $\mathrm{CO}(6-5)$ line emission of the central core (radius $=00^{\prime \prime} 125=$ $52 \mathrm{pc}$ ). The flux is scaled up by a factor of seven. Panel (d): zoom-in plot of the bottom part of panel (c). The arrow marks the location of the $\mathrm{H}^{13} \mathrm{CN}(8-7)$ line with the velocity $v=5745 \mathrm{~km} \mathrm{~s}^{-1}$.

\& Henkel 1991; Nakajima et al. 2011; Sakamoto et al. 2013). Of particular interest is detection of the $\mathrm{H}^{13} \mathrm{CN}$ (4-3) line, with a flux of $f_{\mathrm{H}^{13} \mathrm{CN}(4-3)}=40.8 \pm 9.4 \mathrm{Jy} \mathrm{km} \mathrm{s}^{-1}$, in the nuclear region of NGC 4418 (Sakamoto et al. 2013). Similar to NGC 34, NGC 4418 is also a local LIRG with a putative obscured AGN. Assuming $f_{\mathrm{H}^{13} \mathrm{CN}(8-7)} / f_{\mathrm{H}^{13} \mathrm{CN}(4-3)}=f_{\mathrm{HCN}(8-7)} / f_{\mathrm{HCN}(4-3)}$ $\left(\mathrm{H}^{13} \mathrm{CN}\right.$ lines and HCN lines have nearly identical $T_{\mathrm{ex}}$ and $\left.n_{\text {crit }}\right)$, and the ratio $f_{\mathrm{HCN}(8-7)} / f_{\mathrm{HCN}(4-3)}=0.85 \pm 0.20$ found in the Galactic center circumnuclear disk by Mills et al. (2013) is

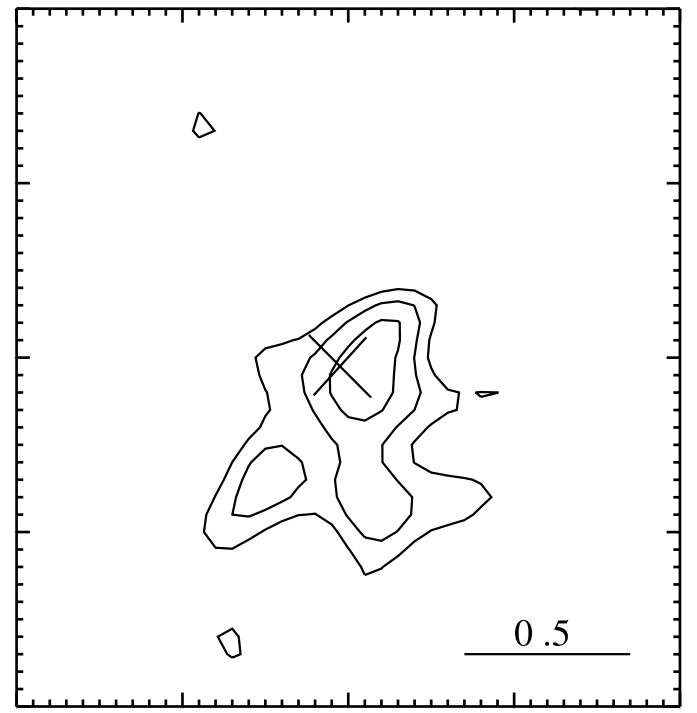

Figure 7. Contour map of the redshifted feature (integrated over channels in velocity range of $6000 \mathrm{~km} \mathrm{~s}^{-1}<v<6200 \mathrm{~km} \mathrm{~s}^{-1}$ ). The contour levels are $\sigma \times[3,4,5]$, and $\sigma=0.46 \mathrm{Jy}_{\text {beam }}^{-1} \mathrm{~km} \mathrm{~s}^{-1}$ (rms). The central cross marks the position of the $\mathrm{CO}(6-5)$ line emission peak, and lengths of the two axes of the cross represent the major and minor axes of the ALMA beam. The map is $2^{\prime \prime} \times 2^{\prime \prime}$ in size.

applicable to NGC 4418 , we can predict that $f_{\mathrm{H}^{13} \mathrm{CN}(8-7)}=$ $34.7 \pm 11.4 \mathrm{Jy} \mathrm{km} \mathrm{s}^{-1}$ for NGC 4418. Dividing this by its $\mathrm{CO}(6-5)$ flux measured by Herschel FTS, $f_{\mathrm{CO}(6-5)}=1068 \pm$ $116 \mathrm{Jy} \mathrm{km} \mathrm{s}^{-1}$ (Lu et al. 2014), we obtain a predicted ratio of $f_{\mathrm{H}^{13} \mathrm{CN}(8-7)} / f_{\mathrm{CO}(6-5)}=0.022 \pm 0.007$ for NGC 4418. This is in very good agreement with the ratio observed for NGC 34, lending support for the hypothesis that the emission feature in the $\mathrm{CO}$ (6-5) profile of NGC 34 is associated with the $\mathrm{H}^{13} \mathrm{CN}(8-7)$ line. Indeed, using models of Meijerink et al. (2011), we found that the observed flux ratio can be reproduced with the following reasonable parameters: (1) turbulent line width $\delta v_{\text {turb }}=2.7 \mathrm{~km} \mathrm{~s}^{-1}$, (2) CO column density $N_{\mathrm{CO}}=$ $10^{18} \mathrm{~cm}^{-2}$, (3) temperature $T>100 \mathrm{~K}$, (4) $\mathrm{H}_{2}$ density $n_{\mathrm{H}_{2}}>$ $10^{3.5} \mathrm{~cm}^{-2}$, (5) column density ratio $N_{\mathrm{H}^{13} \mathrm{CN}} / N_{\mathrm{CO}}>10^{-3}$. Some of these model predictions are presented in Figure 8. High $\mathrm{HCN} / \mathrm{CO}$ ratios are typical for warm molecular gas. As shown by Meijerink et al. (2011), photon-dominated region (PDR) models with strong mechanical heating can produce both high temperatures and high $\mathrm{HCN} / \mathrm{CO}$ ratios, while heating molecular gas to high temperatures with $\mathrm{X}$-rays does not produce high $\mathrm{HCN} / \mathrm{CO}$ ratios.

Another possibility is that the emission feature is associated with an outflow of warm dense gas with a mean velocity of $400 \mathrm{~km} \mathrm{~s}^{-1}$ and maximum velocity of $600 \mathrm{~km} \mathrm{~s}^{-1}$. The contour map of the feature (Figure 7) shows an extended and lopsided morphology. This seems to be consistent with an outflow, although the blueshifted component is missing. Schweizer \& Seitzer (2007) found a high speed neutral gas outflow of $\delta v_{\text {max }}=1050 \mathrm{~km} \mathrm{~s}^{-1}$ in the nuclear region of NGC 34, as revealed by broad blueshifted $\mathrm{Na}$ I $\mathrm{D}$ lines. In recent JVLA and CARMA observations, Fernandez et al. (2014) did not detect any outflow in the nuclear region of NGC 34 in $\mathrm{HI}_{\mathrm{I}}$ or in $\mathrm{CO}(1-0)$, and they set an upper limit for the $\mathrm{CO}$ mass outflow of $40 M_{\odot} \mathrm{yr}^{-1}$ (adopting the CO-to-gas-mass conversion factor for ULIRGs). Assuming an outflow gas with the same $M_{\text {gas }} / L_{\mathrm{CO}(6-5)}$ ratio as that for the static gas (see Section 3.3), a radius of the outflow region of $r=200 \mathrm{pc}$, 

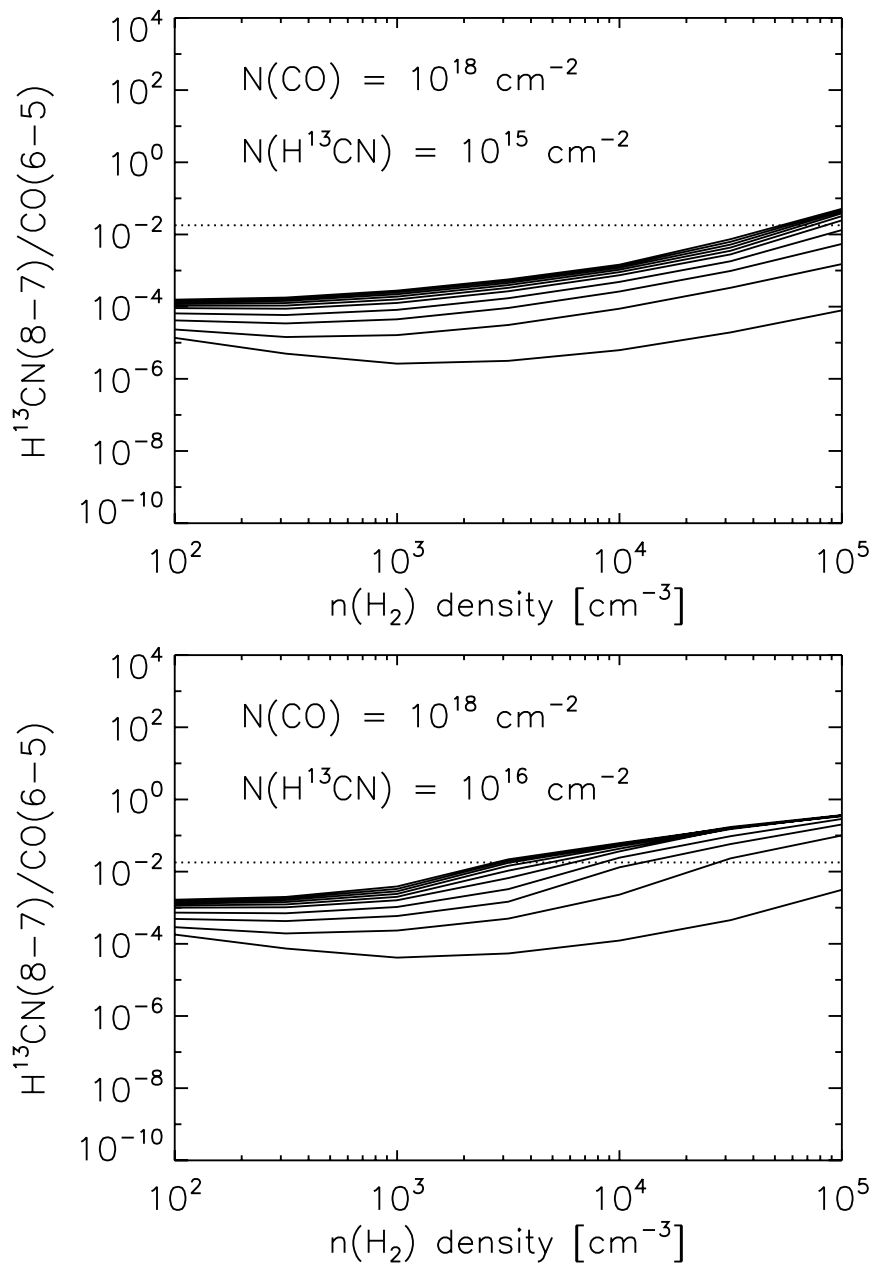

Figure 8. Model predictions for the $f_{\mathrm{H}^{13} \mathrm{CN}(8-7)} / f_{\mathrm{CO}(6-5)}$ ratio. Solid curves present results with different gas temperatures of $T=20,40, \ldots, 200 \mathrm{~K}$. The dotted line marks the observed flux ratio between the redshifted feature and the CO (6-5) line.

a mean outflow velocity of $400 \mathrm{~km} \mathrm{~s}^{-1}$, and adopting a ULIRG conversion factor (Downes \& Solomon 1998), we estimate a mass loss rate of $\dot{M}=32 M_{\odot} \mathrm{yr}^{-1}$ from the integrated flux of the feature using the formalism of Rupke et al. (2002). This is consistent with the upper limit set by Fernandez et al. (2014).

We cannot rule out either of the two scenarios, and the feature in the redshifted wing of $\mathrm{CO}(6-5)$ profile can be due to the $\mathrm{H}^{13} \mathrm{CN}$ line emission or/and an outflow. Observations of other molecular lines with high resolution and high sensitivity are required to resolve this puzzle.

The spectrum of the central unresolved core, measured using an aperture of $r=0^{\prime \prime} 125$, is shown in the panel (c) of Figure 6. Compared to the spectrum of the entire central gas disk, the mean and the FWHM are nearly identical but the double-horn shape is less symmetric. The red peak is significantly more prominent than the blue peak. Different from the large aperture spectrum in panel (a), the spectrum in the core shows a weak blueshifted wing between $5250 \leqslant v \leqslant 5450 \mathrm{~km} \mathrm{~s}^{-1}$. In Figure 9, we present model fitting to the core spectrum using a simple rotating ring model. In order to account for the asymmetric distribution of the spectrum, a density gradient along the major axis of the projected ring is introduced, with the ratio of $\rho_{\max } / \rho_{\min }$ being a free parameter. The least-squares fitting results for the model parameters are as follows: (1) the central velocity of the ring: $v_{\text {center }}=5691 \pm 18 \mathrm{~km} \mathrm{~s}^{-1}$; (2) the projected rotation velocity

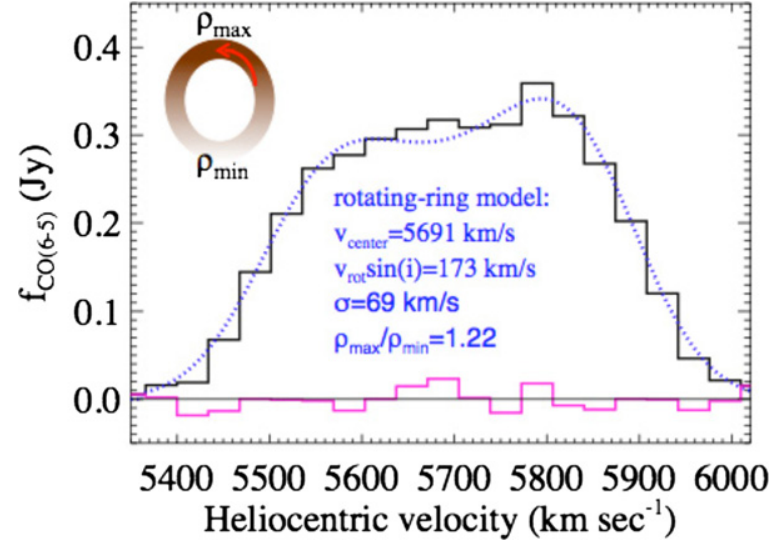

Figure 9. Model fit of the spectrum of the unresolved core. The black histogram presents the data and the blue dotted line the model. The pink histogram near the bottom of the plot presents the residual. The model assumes a rotating ring with a central velocity of $v_{\text {center }}=5691 \mathrm{~km} \mathrm{~s}^{-1}$, a projected rotation velocity of $v_{\text {rot }} \sin (i)=173 \mathrm{~km} \mathrm{~s}^{-1}$, and a velocity dispersion of $\sigma=69 \mathrm{~km} \mathrm{~s}^{-1}$. In order to account for asymmetric distribution of the spectrum, the model also assumes a density gradient along the major axis of the projected ring, with the $\rho_{\max } / \rho_{\min }=1.22$.

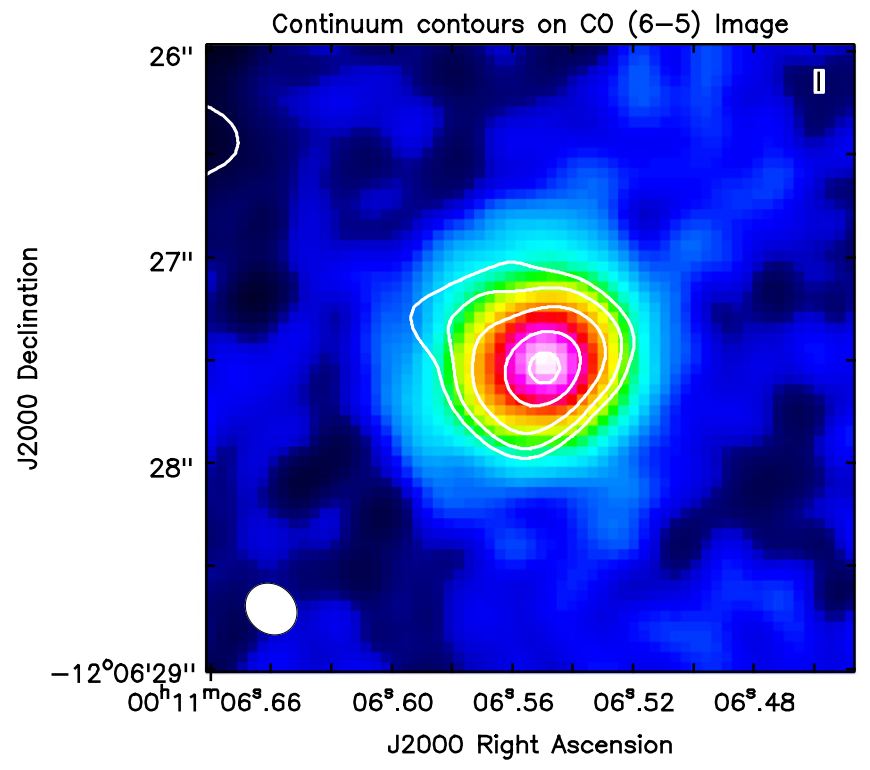

Figure 10. Contours of the dust continuum at $435 \mu \mathrm{m}$ overlaid on the $\mathrm{CO}(6-5)$ image. The contour levels are $[1,2,4,8,12] \times 5.7$ mJy beam $^{-1}$.

of the ring: $v_{\text {rot }} \sin (i)=173 \pm 1 \mathrm{~km} \mathrm{~s}^{-1}$ (we did not attempt to constrain the inclination angle $i$ separately); (3) the intrinsic (Gaussian) velocity dispersion: $\sigma=69 \pm 7 \mathrm{~km} \mathrm{~s}^{-1}$; and (4) the ratio between the maximum and minimum density in the ring: $\rho_{\max } / \rho_{\min }=1.22 \pm 0.30$. This simple model gives a reasonably good fit to the data including the blue-wing. Other models, including a model with two separate Gaussians and another model with a rotating ring plus a Gaussian, can fit the data equally well but with more parameters.

\subsection{Dust Continuum Emission}

A contour map of the continuum at $435 \mu \mathrm{m}$ is overlaid on the CO (6-5) image in Figure 10. The centers of the two maps coincide very well with each other. The continuum was detected only in the inner part of the CO (6-5) disk, and is slightly elongated with a P.A. $=315^{\circ}$. Both the size and the 


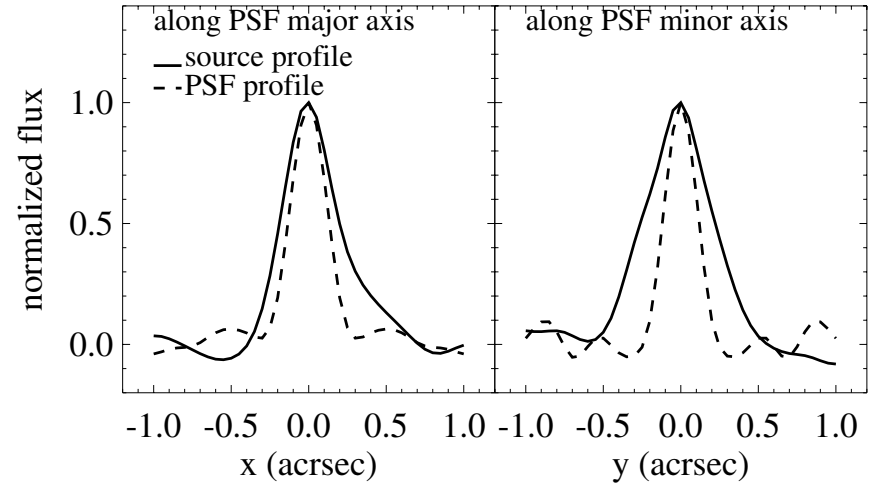

Figure 11. Comparisons of profiles of the $435 \mu \mathrm{m}$ dust continuum emission and the synthesized beam.

orientation of the continuum emission region agree well with those of the "nuclear bulge" found in the HST V-band image by Schweizer \& Seitzer (2007), which is also visible as a "cusp" in the $H$ band (Haan et al. 2013). Indeed, there is a hint that the CO (6-5) line emission may have a central component corresponding to the same nuclear bulge, plus a more extended component (undetected in the continuum) that corresponds to the $V$-band nuclear disk. There is an interesting continuum feature, extending $\sim 0$. 7 toward the northeast direction from the center, which has no correspondence in the $\mathrm{CO}(6-5)$ map, nor in high resolution HST maps. Like the line emission, the continuum is also well resolved by the ALMA beam (Figure 11), with a peak surface density of $76.2 \pm 11.4 \mathrm{mJy}^{\text {beam }}{ }^{-1}$ (peak brightness temperature $=3.9 \pm 0.6 \mathrm{~K}$ ) and an FWHM size of 0.5 . The total flux density of the continuum is $f_{435 \mu \mathrm{m}}=275 \pm 41 \mathrm{mJy}$. NGC 34 was observed by Herschel-SPIRE (Griffin et al. 2010) both in the photometry mode (J. Chu et al., in preparation) and in the FTS mode (P. van der Werf et al., in preparation). Because the error of the continuum measured in the FTS mode is large $(\sim 1 \mathrm{Jy})$, we estimated the total flux of the $435 \mu \mathrm{m}$ continuum of NGC 34 by interpolating SPIRE photometer fluxes $f_{350 \mu \mathrm{m}, \text { SPIRE }}=1095 \mathrm{mJy}$ and $f_{500 \mu \mathrm{m}, \mathrm{SPIRE}}=303 \mathrm{mJy}$, and found $f_{435 \mu \mathrm{m}, \text { SPIRE }}=517 \mathrm{mJy}$. The ratio between $f_{435 \mu \mathrm{m}}$ and $f_{435 \mu \mathrm{m} \text {,SPIRE }}$ then yields an interferometer-to-single-dish flux ratio of $0.53 \pm 0.08$. This indicates a diffuse component much more extended than the central disk, contributing up to $\sim 50 \%$ of the cold dust emission in NGC 34.

\subsection{Gas Mass and Dust Mass}

Fernandez et al. (2014) recently observed the CO (1-0) emission of NGC 34 using CARMA with a synthesized beam of 2 . $48 \times 2$. 12 . The integrated flux in the central beam of the $\mathrm{CO}(1-0)$ map is $f_{\mathrm{CO}(1-0)}=42 \pm 4 \mathrm{Jy} \mathrm{km} \mathrm{s}^{-1}$, corresponding to a $\mathrm{CO}(1-0)$ luminosity of $L_{\mathrm{CO}(1-0)}=(7.1 \pm$ $0.7) \times 10^{8} \mathrm{~K} \mathrm{~km} \mathrm{~s}^{-1} \mathrm{pc}^{-2}$. Assuming that this $\mathrm{CO}(1-0)$ luminosity is concentrated in the central disk detected by ALMA, which has a physical size comparable to that of the CARMA beam ( $\sim 800 \mathrm{pc})$, and adopting a Galactic conversion factor $\alpha_{\mathrm{CO}}=4.3 M_{\odot}\left(\mathrm{K} \mathrm{km} \mathrm{s}^{-1} \mathrm{pc}^{2}\right)^{-1}$ (Bolatto et al. 2013), the gas mass in the central disk is estimated to be $M_{\text {gas }}=10^{9.49 \pm 0.04} M_{\odot}$. If instead adopting the conversion factor advocated by Downes \& Solomon (1998) for ULIRGs, $\alpha_{\mathrm{CO}}=0.8 M_{\odot}\left(\mathrm{K} \mathrm{km} \mathrm{s}^{-1} \mathrm{pc}^{2}\right)^{-1}$, a much lower gas mass is found: $M_{\mathrm{gas}}=10^{8.76 \pm 0.04} M_{\odot}$.
The dust mass can be estimated from the continuum flux using

$$
\begin{aligned}
M_{\mathrm{dust}}= & \frac{c^{2} f_{435 \mu \mathrm{m}} D^{2}}{2 v^{2} k T \kappa_{690 \mathrm{GHz}}} \\
= & 9.2 \times 10^{6} M_{\odot}\left(\frac{f_{435 \mu \mathrm{m}}}{275 \mathrm{mJy}}\right)\left(\frac{D}{84 \mathrm{Mpc}}\right)^{2} \\
& \times\left(\frac{T}{43.0 \mathrm{~K}}\right)^{-1}\left(\frac{\kappa_{690 \mathrm{GHz}}}{0.16 \mathrm{~m}^{2} \mathrm{~kg}^{-1}}\right)^{-1}
\end{aligned}
$$

where $\kappa_{690 \mathrm{GHz}}$ is the mass-opacity at $690 \mathrm{GHz}$ (central frequency of the continuum), for which we assumed $\kappa_{690 \mathrm{GHz}}=$ $\kappa_{1200 \mathrm{GHz}} \times(690 / 1200)^{2}$ and $\kappa_{1200 \mathrm{GHz}}=0.48 \mathrm{~m}^{2} \mathrm{~kg}^{-1}$ (Dale et al. 2012). The dust temperature, $T=43.0 \mathrm{~K}$, is estimated using the formalism of Scoville (2013) for extremely obscured nuclear starbursts (i.e., optically thick for the IR emission of $\left.\lambda \lesssim 100 \mu \mathrm{m}^{14}\right): T_{\text {dust }}=630 \times\left[L_{\mathrm{IR}} / 10^{12} L_{\odot}\right]^{1 / 4} / R_{\mathrm{pc}}^{1 / 2} \mathrm{~K}$. Here, we assume the radius of the dusty region is $R_{\mathrm{pc}}=100$, and $70 \%$ of the IR luminosity of NGC 34 is inside this radius (as hinted by the high resolution radio continuum data). Changing the fraction to $50 \%$ or $100 \%$ will make a difference in the dust temperature of $\pm 4 \mathrm{~K}$, corresponding to a $10 \%$ error. Given the $30 \%$ error for $\kappa$ (James et al. 2002), 15\% flux calibration error, and $\sim 10 \%$ error in the temperature estimate, the dust mass is $M_{\text {dust }}=10^{6.97 \pm 0.13} M_{\odot}$.

\section{COMPARISONS WITH PREVIOUS RESULTS}

NGC 34 is a late stage merger with prominent tidal tails (Mazzarella \& Boroson 1993; Schweizer \& Seitzer 2007). Schweizer \& Seitzer (2007) carried out a detailed analysis of NGC 34 and their results are consistent with a merger of two gas-rich galaxies with mass ratio of $m / M \sim 1 / 2-1 / 3$. The disks of two galaxies are already coalesced. An early MIR observation of Miles et al. (1996) found evidence for a second nucleus about 1".2 south of the primary nucleus, which might coincide with a weak radio source in the high resolution Very Large Array (VLA) radio continuum map (Condon et al. 1991). However, such a source was not found in the HST NICMOS $K$-band and $H$-band images (Schweizer \& Seitzer 2007; Haan et al. 2011). In our ALMA observations, we did not detect this source in the $\mathrm{CO}(6-5)$ line emission or in the dust continuum, either. Therefore, our observations are in good agreement with the NICMOS observations and is consistent with a separation limit of $\lesssim 50$ pc for any possible double nuclei configuration.

The most solid evidence for the existence of an AGN in NGC 34 is found in the X-ray (Guainazzi et al. 2005; Shu et al. 2007; Brightman \& Nandra 2011; Esquej et al. 2012). Recent XMM-Newton observations (Brightman \& Nandra 2011; Esquej et al. 2012) found an X-ray luminosity of $L_{X, 2-10 \mathrm{keV}}=$ $1.4_{0.2}^{+0.4} \times 10^{42} \mathrm{erg} \mathrm{s}^{-1}$, after the correction for absorption by a neutral gas column density of $N_{\mathrm{H}}=7.5_{-2.1}^{+3.1} \times 10^{23} \mathrm{~cm}^{-2}$. Such a high X-ray luminosity cannot be explained by a pure starburst of SFR $<200 M_{\odot} \mathrm{yr}^{-1}$ (Ranalli et al. 2003).

Any possible contribution from the AGN to the $\mathrm{CO}(6-5)$ line emission and the continuum emission should be inside the unresolved core (radius $=50 \mathrm{pc}$ ). For the line emission, the ratio of core flux and the total flux of the central disk is $f_{\mathrm{CO}(6-5) \text {, core }} / f_{\mathrm{CO}(6-5)}=19 \%$, and for the continuum $f_{435 \mu \mathrm{m}, \text { core }} / f_{435 \mu \mathrm{m}}=28 \%$. Hence, even if the AGN would be 14 The gas column density profile shown in Figure 13 confirms that indeed the
central dusty region is optically thick at $\lambda \lesssim 100 \mu \mathrm{m}$. 


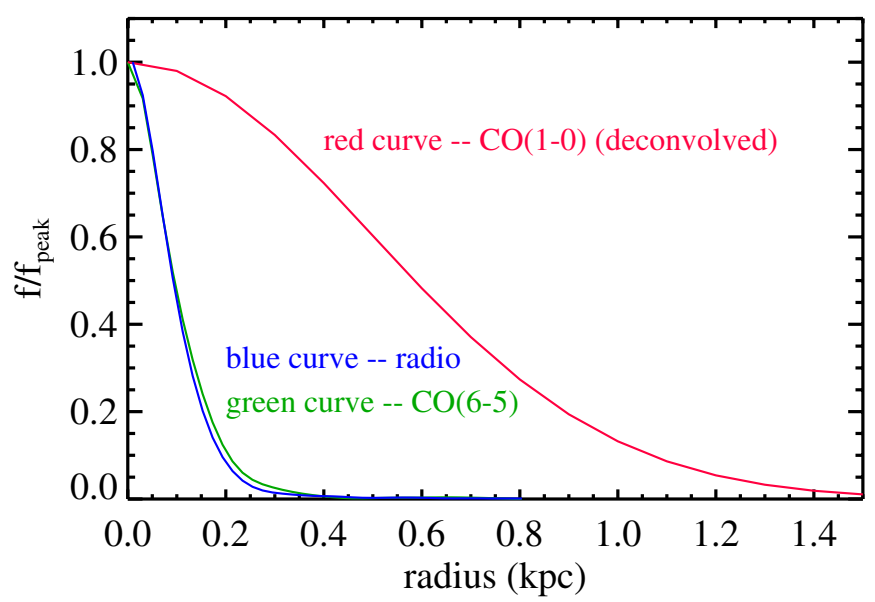

Figure 12. Comparisons of normalized radial profiles of the $\mathrm{CO}(6-5)$, radio continuum (Condon et al. 1991), and PSF-deconvolved CO (1-0) (Fernandez et al. 2014).

fully responsible for the heating of these "cores," one could still conclude that the AGN contribution to total emission in both cases is insignificant. This is consistent with previous studies that found low AGN contribution (in the range of 1\%-10\%) to the total IR emission of NGC 34 (Prouton et al. 2004; Vega et al. 2008; Esquej et al. 2012; Murphy et al. 2013; Stierwalt et al. 2013). The VLBI observations of Lonsdale et al. (1993) did not detect any unresolved core. This sets an upper limit for the core flux of the radio continuum at $18 \mathrm{~cm}$ of $S_{\text {core }}<2.5 \mathrm{mJy}$, suggesting that the AGN contributes $\lesssim 5 \%$ of the total radio continuum flux.

The star formation in NGC 34 is concentrated in a nuclear starburst, which dominates the IR and radio luminosities of the LIRG (Condon et al. 1991; Miles et al. 1996; Gorjian et al. 2004; Esquej et al. 2012). Esquej et al. (2012) estimated the size of the starburst region is $\sim 0.5-1 \mathrm{kpc}$ (corresponding to $1^{\prime \prime} .25-2$.'.5 FWHM), and did not find any significant IR emission beyond $2 \mathrm{kpc}\left(5^{\prime \prime}\right.$ FWHM). Our higher angular resolution ALMA maps revealed a central disk of FWHM $=0.5(0.2 \mathrm{kpc})$, significantly smaller than the MIR emitting region studied by Miles et al. (1996) and Esquej et al. (2012). In Figure 12, we compared the radial profiles of the $\mathrm{CO}(6-5)$ and radio continuum at $8.44 \mathrm{GHz}$ (beam $=0.39 \times 0.29$, Condon et al. 1991) and found very good agreement. ${ }^{15}$ Since the AGN contribution to the radio continuum is negligible (Lonsdale et al. 1993), this suggests a tight correlation between the warm gas probed by the $\mathrm{CO}(6-5)$ and current star formation activity probed by the radio continuum on the time scale of $\sim 10^{8} \mathrm{yr}(\mathrm{Xu} 1990)$. It is unlikely that any substantial fraction of the radio continuum is missing in the high resolution $8.44 \mathrm{GHz}$ map because the total flux obtained using the map fits very well in the spectral index analyses (Clemens et al. 2008; Fernandez et al. 2014), together with radio fluxes in other bands of much lower resolutions (e.g., $f_{1.4 \mathrm{GHz}}$ with beam size of $45^{\prime \prime}$; Condon et al. 1998). The larger size of the MIR emission could be due to contributions of older stars (e.g., those in the central disk of $\sim 4 \times 10^{8} \mathrm{yr}$ old; Schweizer $\&$ Seitzer 2007) to the polycyclic aromatic hydrocarbon (PAH) emission (Lu et al. 2003).

The CO (6-5) disk is also significantly more compact than the CO (1-0) disk found by Fernandez et al. (2014); after deconvolving with a Gaussian beam of 2". $48 \times 2$ ". 14 (Fernandez

\footnotetext{
15 There is a position offset of $\sim 0^{\prime \prime} .1$ between peak of the CO (6-5) and that of the radio continuum, which we attributed to the astrometrical error of the ALMA observations.
}

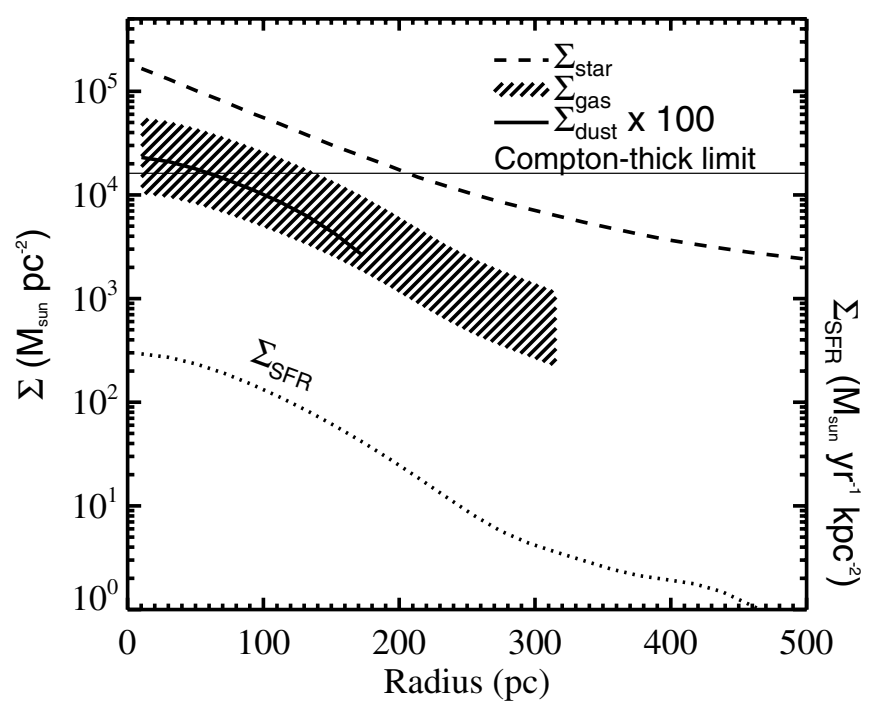

Figure 13. Radial profiles of column densities of stars, gas, and dust (multiplied by a factor of 100). The stellar mass is estimated using the HST NICMOS $H$-band data (Haan et al. 2011). The upper boundary of the stripe representing the gas mass column density profile is estimated from ALMA CO (6-5) observations using the Galactic conversion factor (Bolatto et al. 2013) and the lower boundary the ULIRG conversion factor (Downes \& Solomon 1998). The dust mass column density is derived from ALMA $435 \mu \mathrm{m}$ continuum observations. The Comptonthick limit corresponds to a gas column density of $N_{\mathrm{H}}=1.5 \times 10^{24} \mathrm{~cm}^{-2}$.

et al. 2014), the CO (1-0) emission has a half-peak radius of $0.6 \mathrm{kpc}$, which is about 6 times of that of the $\mathrm{CO}(6-5)$ emission (Figure 12). It appears that a large fraction of the $\mathrm{CO}(1-0)$ emission is not associated with current star formation activity (as indicated by the radio continuum). This is consistent with previous studies which suggested that the low J CO luminosities may be dominated by the emission of diffuse gas not closely related to the active star formation regions (Scoville et al. 1997; Downes \& Solomon 1998; Bryant \& Scoville 1999; Gao et al. 2001). The SMA CO (3-2) observations have also shown more compact emission regions (Wilson et al. 2008; Ueda et al. 2012; Sakamoto et al. 2013) and closer correlation with the star formation activity compared to the $\mathrm{CO}(1-0)$ emission (Yao et al. 2003; Iono et al. 2004; Wang et al. 2004; Iono et al. 2009). Probing even higher density gas than the CO (3-2) line does, the $\mathrm{CO}(6-5)$ emission has perhaps more in common with dense molecular lines such as HCN $(1-0)\left(n_{\text {crit }}=2.6 \times 10^{6}\right)$, for which Gao \& Solomon (2004) found a very tight and linear correlation with the SFR.

In Figure 13, the radial profiles of the gas and dust column densities are presented. These were estimated using radial profiles of the $\mathrm{CO}(6-5)$ and the continuum and assuming constant mass-to-luminosity ratios. Here we adopted, according to values listed in Table 2 , an $M_{\mathrm{gas}} / L_{\mathrm{CO}(6-5)}$ ratio of 5.3 (1.0) $M_{\odot}\left(\mathrm{K} \mathrm{km} \mathrm{s}^{-1} \mathrm{pc}^{2}\right)^{-1}$ for Galactic (ULIRG) conversion factor and an $M_{\text {dust }} / L_{435 \mu \mathrm{m}}$ ratio of $0.022 M_{\odot} L_{\odot}^{-1}$. The plot shows very high gas column density in the inner most region of $r<100$ pc: the peak $\Sigma_{\text {gas }}$ derived using the Galactic conversion factor is about 2.5 times above the Compton-thick $\operatorname{limit}\left(N_{\mathrm{H}}=1.5 \times 10^{24} \mathrm{~cm}^{-2}\right)$, and that derived using the ULIRG conversion factor is only a factor of two lower than the Comptonthick limit. It is worth noting that the central gas column density estimated using ALMA data is consistent with that estimated using the X-ray data (Brightman \& Nandra 2011; Esquej et al. 2012).

For comparison, we also plot in Figure 13 the $\Sigma_{\text {SFR }}$ profile estimated using the radio continuum at $8.44 \mathrm{GHz}$ and the $\Sigma_{\text {star }}$ 
Table 2

$\mathrm{CO}(6-5)$ and $435 \mu \mathrm{m}$ Continuum in NGC 34

\begin{tabular}{lcccccccccc}
\hline \hline & $(1)$ & $(2)$ & $(3)$ & $(4)$ & $(5)$ & $(6)$ & $(7)$ & $(8)$ & $(9)$ & $(10)$ \\
\hline Emission & Size & $b / a$ & P.A. & $\delta v(\mathrm{FWHM})$ & $T_{\text {peak }}$ & $f_{\text {ALMA }}$ & $f_{\text {ALMA }} / f_{\text {Hsch }}$ & $\log (L)$ & $\log (M)$ & $\log \left(\Sigma_{\text {peak }}\right)$ \\
\hline & $(\mathrm{pc})$ & & $(\mathrm{deg})$ & $\left(\mathrm{km} \mathrm{s}^{-1}\right)$ & $(\mathrm{K})$ & $\begin{array}{c}(\mathrm{Jy} \mathrm{km} \mathrm{s}) \\
\left(\mathrm{mJy}^{-1}\right)\end{array}$ & & $\begin{array}{c}\left(\mathrm{K} \mathrm{km} \mathrm{s}^{-1} \mathrm{pc}^{2}\right) \\
\left(L_{\odot}\right)\end{array}$ & $\left(M_{\odot}\right)$ & $\left(M_{\odot} \mathrm{pc}^{-2}\right)$ \\
& & & & & & & & \\
\hline CO (6-5) & 200 & $\sim 0.9$ & 345 & 406 & $36.8 \pm 5.5$ & $1004 \pm 151$ & $1.07 \pm 0.18$ & $8.68 \pm 0.06$ & $9.13 \pm 0.36$ & $4.37 \pm 0.36$ \\
Continuum & 200 & $\sim 0.7$ & 315 & & $3.9 \pm 0.6$ & $275 \pm 41$ & $0.53 \pm 0.08$ & $8.63 \pm 0.06$ & $6.97 \pm 0.13$ & $2.36 \pm 0.13$
\end{tabular}

Notes. Column 1: size (FWHM) of the emission region; 2: minor-to-major axis ratio; 3: position angle; 4: FWHM of the velocity distribution; 5: peak brightness temperature; 6: for the $\mathrm{CO}(6-5)$ : integrated flux in $\mathrm{Jy} \mathrm{km} \mathrm{s}^{-1}$, for the continuum: flux density in mJy; 7: ratio between the flux measured by ALMA (interferometer) and by Herschel (single dish); 8: for the CO (6-5): logarithm of the CO (6-5) luminosity in $\mathrm{K} \mathrm{km} \mathrm{s}^{-1} \mathrm{pc}^{2}$, for the continuum: logarithm of the $435 \mu \mathrm{m}$ continuum luminosity in $L_{\odot}$; 9: for the CO (6-5): logarithm of the gas mass, which is the geometrical mean of two estimations based on the Galactic conversion factor (Bolatto et al. 2013) and the ULIRG conversion factor (Downes \& Solomon 1998), respectively, and the error corresponds to the half difference between the two; for the continuum: logarithm of the dust mass; 10: for the CO (6-5): logarithm of the peak column density of the gas mass, again an average of the two estimations based on the Galactic conversion factor and the ULIRG conversion factor, respectively; for the continuum: logarithm of the peak dust column density.

profile derived using the $H S T$ NICMOS $H$-band data (angular resolution: 0'.15; Haan et al. 2011). In order to convert $P_{8.44 \mathrm{GHz}}$ to the SFR, we assumed that $S_{1.4 \mathrm{GHz}} / S_{8.44 \mathrm{GHz}}=3.86$ (Condon et al. 1991), $q=\log \left(L_{\mathrm{fir}} /\left(3.75 \times 10^{12} \mathrm{~Hz}\right) / P_{1.4 \mathrm{GHz}}\right)=2.34$ (Yun et al. 2001), $L_{\mathrm{IR}}(8-1000 \mu \mathrm{m}) / L_{\mathrm{fir}}(40-120 \mu \mathrm{m})=1.8$, and $\mathrm{SFR} /\left(M_{\odot} \mathrm{yr}^{-1}\right)=L_{\mathrm{IR}} /\left(1.16 \times 10^{10} L_{\odot}\right)($ Kennicutt 1998a; converted to a Kroupa initial mass function (IMF)). For the estimate of $\Sigma_{\text {star, }}$, we assumed that the stellar mass in the inner most $\sim 500 \mathrm{pc}$ of NGC 34 is dominated by the starburst, as suggested by the simulation results of Hopkins et al. (2008b), and adopted a constant $H$-band luminosity-tomass ratio predicted by a single-burst stellar population model of Bruzual \& Charlot (2003) with the Kroupa IMF, solar metallicity, and starburst age of $\tau=4 \times 10^{8} \mathrm{yr}$ (Schweizer \& Seitzer 2007). An $H$-band extinction correction of $A_{\mathrm{H}}=0.46$ mag was applied, derived from the $\mathrm{Pa} \beta$-to- $\mathrm{Br} \gamma$ ratio (RodriguezArdila et al. 2005) and the extinction curve of Calzetti et al. (2000). ${ }^{16}$ Interestingly, the star formation timescale derived from $\Sigma_{\text {star }} / \Sigma_{\text {SFR }}$ ratio is rather constant in the inner part of the disk of $r \lesssim 200 \mathrm{pc}$, with a value of $\sim 8 \times 10^{8} \mathrm{yr}$. Given the uncertainty of $\Sigma_{\text {star }}$, which is on the order of a factor of two (due to errors in both the assumed light-to-mass ratio and the extinction correction), this is marginally higher than the starburst age ( $\left.\tau=4 \times 10^{8} \mathrm{yr}\right)$ found by Schweizer \& Seitzer (2007) using the optical colors, consistent with a relatively old starburst already passed its peak activity. Indeed, the comparison between $\Sigma_{\mathrm{SFR}}$ and $\Sigma_{\text {gas }}$ yields a gas exhaustion time scale of $M_{\text {gas }} / \mathrm{SFR} \sim 1.6(0.3) \times 10^{8} \mathrm{yr}$ if the Galactic (ULIRG) conversion factor is used, which is significantly shorter than the star formation time scale. Nevertheless, the starburst is still very strong. With $\Sigma_{\mathrm{SFR}} \simeq 300 M_{\odot} \mathrm{yr}^{-1} \mathrm{kpc}^{-2}$ and $\Sigma_{\text {gas }} \simeq 2 \times 10^{4} M_{\odot} \mathrm{pc}^{-2}$ at the central peak, it falls right on the Kennicutt-Schimdt law and is among the extreme starbursts with the highest $\Sigma_{\text {SFR }}$ and $\Sigma_{\text {gas }}$ (see Figure 6 of Kennicutt 1998b; but also see Liu \& Gao 2012).

\section{SUMMARY}

The CO (6-5) line emission in the central region of NGC 34 (radius $\lesssim 500 \mathrm{pc}$ ) is well resolved by the ALMA beam

\footnotetext{
$\overline{16}$ The $\Sigma_{\text {dust }}$ plotted in Figure 13, if uniformly distributed in a foreground screen, indicates much higher $A_{\mathrm{H}}$. However, it is likely that the dust is clumpy and much of the nuclear bulge is in front of dust.
}

$(0,26 \times 0.23)$. This is the first time the warm and dense gas in the nuclear region of an LIRG is resolved with a linear resolution of $\sim 100 \mathrm{pc}$. Both the morphology and kinematics of the CO (6-5) line emission are rather regular, consistent with a compact rotating disk which has an FWHM size of 200 pc and extends to a radius of $r=320 \mathrm{pc}$. The integrated $\mathrm{CO}(6-5)$ line flux of the disk is $f_{\mathrm{CO}(6-5)}=1004( \pm 151) \mathrm{Jy} \mathrm{km} \mathrm{s}{ }^{-1}$, recovering all the single dish CO (6-5) line flux of NGC 34 measured by Herschel. The line profile shows a double-horn shape, with an FWHM of $406 \mathrm{~km} \mathrm{~s}^{-1}$.

A significant emission feature is detected on the redshifted wing of the profile, coincident with the frequency of the $\mathrm{H}^{13} \mathrm{CN}$ (8-7) line emission (rest-frame frequency = $690.552 \mathrm{GHz}$ ), with an integrated flux of $17.7 \pm 2.1$ (random) \pm 2.7 (sysmatic) $\mathrm{Jy} \mathrm{km} \mathrm{s}^{-1}$. However, it cannot be ruled out that the feature is due to an outflow of warm dense gas with a mean velocity of $400 \mathrm{~km} \mathrm{~s}^{-1}$.

The $435 \mu \mathrm{m}$ continuum emission is resolved into an elongated configuration (P.A. $=315^{\circ}$ ). The flux is $f_{435 \mu \mathrm{m}}=$ $275( \pm 41)$ mJy $(53 \pm 8 \%$ of the total $435 \mu \mathrm{m}$ flux derived from Herschel observations), corresponding to a dust mass of $M_{\text {dust }}=10^{6.97 \pm 0.13} M_{\odot}$. An unresolved central core (radius $\simeq 50 \mathrm{pc}$ ) contributes $28 \%$ of the continuum flux and $19 \%$ of the CO (6-5) flux detected by ALMA, consistent with insignificant contributions of the AGN to both emissions.

The CO (6-5) disk is a factor of six smaller than the $\mathrm{CO}$ (1-0) disk found by Fernandez et al. (2014). Comparison with radio continuum suggests that the nuclear starburst has about the same distribution of the warm and dense gas probed by $\mathrm{CO}$ (6-5), while much of the diffuse gas probed by $\mathrm{CO}(1-0)$ is not associated with star formation. Both the $\mathrm{CO}(6-5)$ line and continuum distributions indicate a very high gas column density $\left(\gtrsim 10^{4} M_{\odot} \mathrm{pc}^{-2}\right)$ in the nuclear region (radius $\lesssim 100 \mathrm{pc}$ ), consistent with the extremely high SFR density found in the same region.

C.K.X. acknowledges useful discussions with George Privon, Eckard Sturm, Francois Schweizer, and Ximena Fernandez. Kim Scott and Tony Remijan from NAASC are thanked for their help on data reduction. Y.G. is partially supported by NSFC-11173059, NSFC-11390373, and CAS-XDB09000000. Y.Z. thanks the NSF of Jiangsu Province for partial support under grant BK2011888. V.C. acknowledges partial 
support from the EU FP7 grant PIRSES-GA-2012-316788. This paper makes use of the following ALMA data: ADS/ JAO.ALMA\#2011.0.00182.S. ALMA is a partnership of ESO (representing its member states), NSF (USA), and NINS (Japan), together with NRC (Canada) and NSC and ASIAA (Taiwan), in cooperation with the Republic of Chile. The Joint ALMA Observatory is operated by ESO, AUI/NRAO, and NAOJ. This research has made extensive use of the NASA/ IPAC Extragalactic Database (NED), which is operated by the Jet Propulsion Laboratory, California Institute of Technology, under contract with the National Aeronautics and Space Administration.

\section{REFERENCES}

Aalto, S., Garcia-Burillo, S., Muller, S., et al. 2012, A\&A, 537, A44 Armus, L., Heckman, T. M., \& Miley, G. K. 1990, ApJ, 364, 471

Armus, L., Mazzarella, J. M., Evans, A. S., et al. 2009, PASP, 121, 599

Bolatto, A. D., Wolfire, M., \& Leroy, A. K. 2013, ARA\&A, 51, 207

Brightman, M., \& Nandra, K. 2011, MNRAS, 413, 1206

Bruzual, G., \& Charlot, S. 2003, MNRAS, 344, 1000

Bryant, P. M., \& Scoville, N. Z. 1999, AJ, 117, 2632

Calzetti, D., Armus, L., Bohlin, R. C., et al. 2000, ApJ, 533, 682

Carilli, C. L., \& Walter, F. 2013, ARA\&A, 51, 105

Cicone, C., Maiolino, R., Sturm, E., et al. 2014, A\&A, 562, A21

Clemens, M. S., Vega, O., Bressan, A., et al. 2008, A\&A, 477, 95

Combes, F., García-Burillo, S., Casasola, V., et al. 2013, A\&A, 558, A124

Condon, J. J., Cotton, W. D., Greisen, E. W., et al. 1998, AJ, 115, 1693

Condon, J. J., Huang, Z.-P., Yin, Q.-F., \& Thuan, T. 1991, ApJ, 378, 65

Dale, D. A., Aniano, G., Engelbracht, C. W., et al. 2012, ApJ, 745, 95

Downes, D., \& Solomon, P. M. 1998, ApJ, 507, 615

Esquej, P., Alonso-Herrero, A., Perez-Garcia, A. M., et al. 2012, MNRAS, 423,185

Faber, S. M., Willmer, C. N. A., Wolf, C., et al. 2007, ApJ, 665, 265

Fernandez, X., Petric, A. O., Schweizer, F., \& van Gorkom, J. H. 2014, AJ, in press (arXiv:1401.1821)

Feruglio, C., Fiore, F., Piconcelli, E., et al. 2013, A\&A, 558, A87

Feruglio, C., Maiolino, R., Piconcelli, E., et al. 2010, A\&A, 518, L155

Fischer, J., Sturm, E., González-Alfonso, E., et al. 2010, A\&A, 518, L41

Gao, Y., Lo, K. Y., Lee, S.-W., \& Lee, T.-H. 2001, ApJ, 548, 172

Gao, Y., \& Solomon, P. M. 2004, ApJ, 606, 271

Genzel, R., Tacconi, L. J., Rigopoulou, D., Lutz, D., \& Tecza, M. 2001, ApJ, 563,527

Gorjian, V., Werner, M. W., Jarrett, T. H., et al. 2004, ApJ, 605, 156

Griffin, M., Abergel, A., Abreu, A., et al. 2010, A\&A, 518, L3

Guainazzi, M., Matt, G., \& Perola, G. C. 2005, A\&A, 444, 119

Haan, S., Armus, L., Surace, J. A., et al. 2013, MNRAS, 434, 1264

Haan, S., Surace, J. A., Armus, L., et al. 2011, AJ, 141, 100

Harris, A. I., Hills, R. E., Stutozki, J., et al. 1991, ApJ, 382, 75

Heckman, T. M., Armus, L., \& Miley, G. K. 1990, ApJS, 74, 833

Hopkins, P. F., Cox, T. J., Hernquist, L., et al. 2013, MNRAS, 430, 1901

Hopkins, P. F., Cox, T. J., Kere, D., \& Hernquist, L. 2008a, ApJS, 75, 390

Hopkins, P. F., Hernquist, L., Cox, T. J., et al. 2008b, ApJ, 679, 156

Iono, D., Ho, P. T. P., Yun, M. S., et al. 2004, ApJL, 616, L63
Iono, D., Wilson, C. D., Yun, M. S., et al. 2009, ApJ, 695, 1537

James, A., Dunne, L., Eales, S., \& Edmunds, M. G. 2002, MNRAS, 335, 753

Kennicutt, R. C. 1998a, ARA\&A, 36, 189

Kennicutt, R. C. 1998b, ApJ, 498, 541

Kim, D.-C., Veilleux, S., \& Sanders, D. B. 2002, ApJS, 143, 277

Liu, L., \& Gao, Y. 2012, ScChG, 55, 347

Lonsdale, C. J., Smith, H. E., \& Lonsdale, C. J. 1993, ApJL, 405, L9

Lu, N., Helou, G., Werner, M. W., et al. 2003, ApJ, 588, 199

Lu, N., Zhao, Y., Xu, C. K., et al. 2014, ApJ, submitted

Malken, M. A., Gorjian, V., \& Tam, R. 1998, ApJS, 117, 25

Matsushita, S., Iono, D., Petitpas, G. R., et al. 2009, ApJ, 693, 56

Mauersberger, R., \& Henkel, C. 1991, A\&A, 245, 457

Mazzarella, J. M., \& Boroson, T. A. 1993, ApJS, 85, 27

Meijerink, R., Spaans, M., Loenen, A. F., \& van der Werf, P. P. 2011, A\&A, 525, A119

Miles, J. W., Houck, J. R., Hayward, T. L., \& Ashby, M. L. N. 1996, ApJ, 465,191

Mills, E. A. C., Güsten, R., Requena-Torres, M. A., \& Morris, M. R. 2013, ApJ, 779,47

Murphy, E. J., Stierwalt, S., Armus, L., et al. 2013, ApJ, 768, 2

Nakajima, T., Takano, S., Kohno, K., \& Inoue, H. 2011, ApJL, 728, L38

Papadopoulos, P. P., van der Werf, P., Xilouris, E., et al. 2012, MNRAS, 426, 2601

Pilbratt, G. L., Riedinger, J. R., Passvogel, T., et al. 2010, A\&A, 518, L1

Prouton, O. R., Clemens, M., Franceschni, A., et al. 2004, A\&A, 421, 115

Ranalli, P., Comastri, A., \& Setti, G. 2003, A\&A, 399, 39

Rodriguez-Ardila, A., Riffel, R., \& Pastoriza, M. G. 2005, MNRAS, 364, 1041

Rupke, D. S., Veilleux, S., \& Sanders, D. B. 2002, ApJ, 570, 588

Rupke, D. S., Veilleux, S., \& Sanders, D. B. 2005, ApJS, 160, 115

Sakamoto, K., Aalto, S., Costagliola, F., et al. 2013, ApJ, 764, 42

Sanders, D. B., Mazzarella, J. M., Kim, D.-C., Surace, J. A., \& Soifer, B. T. 2003, AJ, 126, 1607

Sanders, D. B., \& Mirabel, I. F. 1996, ARA\&A, 34, 749

Schweizer, F. 1982, ApJ, 252, 455

Schweizer, F., \& Seitzer, P. 2007, AJ, 133, 2132

Scoville, N. Z. 2013, in Secular Evolution of Galaxies, ed. J. Falcón-Barroso \& J. H. Knapen (Cambridge: Cambridge Univ. Press), 491

Scoville, N. Z., Yun, M. S., \& Bryant, P. M. 1997, ApJ, 484, 702

Shu, X. W., Wang, J., Jiang, P., et al. 2007, ApJ, 657, 157

Sliwa, K., Wilson, K. D., Krips, M., et al. 2013, ApJ, 777, 126

Soifer, B. T., Sanders, D. B., Madore, B. F., et al. 1987, ApJ, 320, 238

Stierwalt, S., Armus, L., Surace, J. A., et al. 2013, ApJS, 206, 1

Sturm, E., González-Alfonso, E., Veilleux, S., et al. 2011, ApJL, 733, L16

Toomre, A. 1977, in The Evolution of Galaxies and Stellar Populations, ed.

B. M. Tinsley \& R. B. Larson (New Haven, CT: Yale Univ. Press), 401

Ueda, J., Iono, D., Petitpas, G., et al. 2012, ApJ, 745, 65

Vega, O., Clemens, M. S., Bressan, A., et al. 2008, A\&A, 484, 631

Veilleux, S., Melendez, M., Sturm, E., et al. 2013, ApJ, 776, 27

Wang, J., Zhang, Q., Wang, Z., et al. 2004, ApJL, 616, L67

Wang, J. L., Xia, X. Y., Mao, S., et al. 2006, ApJ, 649, 722

Werner, M. W., Roellig, T. L., Low, F. J., et al. 2004, ApJS, 154, 1

Wilson, C. D., Petitpas, G. R., Iono, D., et al. 2008, ApJS, 178, 189

Wootten, A., \& Thompson, A. R. 2009, IEEE, 97, 1463

Xu, C. 1990, ApJL, 365, L47

Yao, L., Seaquist, E. R., Kuno, N., \& Dunne, L. 2003, ApJ, 588, 771

Yun, M. S., Reddy, N. A., \& Condon, J. J. 2001, ApJ, 554, 803

Zubovas, K., \& King, A. 2012, ApJL, 745, L34 